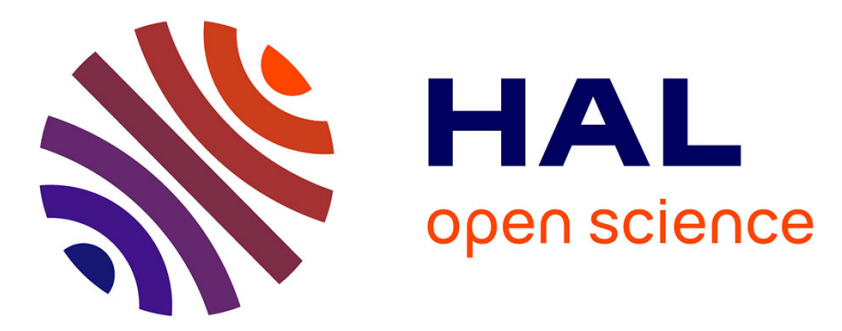

\title{
Dynamic output-feedback controller design for analgesia guided by the pupil size variation
}

\author{
Saïd Zabi, Isabelle Queinnec, Sophie Tarbouriech, Michel Mazerolles
}

\section{To cite this version:}

Saïd Zabi, Isabelle Queinnec, Sophie Tarbouriech, Michel Mazerolles. Dynamic output-feedback controller design for analgesia guided by the pupil size variation. European Control Conference (ECC 2016), Jun 2016, Aalborg, Denmark. pp.2121 - 2126, 10.1109/ECC.2016.7810605 . hal-01474484

\author{
HAL Id: hal-01474484 \\ https://hal.laas.fr/hal-01474484
}

Submitted on 22 Feb 2017

HAL is a multi-disciplinary open access archive for the deposit and dissemination of scientific research documents, whether they are published or not. The documents may come from teaching and research institutions in France or abroad, or from public or private research centers.
L'archive ouverte pluridisciplinaire HAL, est destinée au dépôt et à la diffusion de documents scientifiques de niveau recherche, publiés ou non, émanant des établissements d'enseignement et de recherche français ou étrangers, des laboratoires publics ou privés. 


\title{
Dynamic output-feedback controller design for analgesia guided by the pupil size variation
}

\author{
Said Zabi ${ }^{1}$, Isabelle Queinnec ${ }^{1}$, Sophie Tarbouriech ${ }^{1}$ and Michel Mazerolles ${ }^{2}$
}

\begin{abstract}
This paper pertains to the design of a dynamic output-feedback controller for analgesia taking into account the saturation of the input and the quantization of the output. Actually, the output is given by the smallest magnitude of the stimulus that induces a significant pupil size variation. In addition, to take into account multiple time scale dynamics in the analgesia model, the system is re-expressed by decoupling the fast dynamics from the slow ones.
\end{abstract}

\section{INTRODUCTION}

The goal of clinical anesthesia is to avoid patient awareness (hypnosis) and minimize the response to noxious stimulation (analgesia) through the administration of anesthetic drugs based on clinical indicators [1], [14], [4]. Concerning more specifically the depth of analgesia, several studies have been concerned with the selection of reliable indicators. [7], [2] proposed an evaluation of the depth of analgesia by analyzing the heart rate variability and introduced the ANI (for Autonomic Nervous Index). Blood pressure, grimaces, electrocardiogram (EEG), the difference of entropy and other indicators were also proposed in [10], [15], [11], [23]. In this work we use the response of the pupil as an indicator, being shown that its area variates according to nociceptive stimulation [16]. Actually, [3] showed that the pupil response to a painful stimulus is a better indicator of the effect site remifentanil concentration than haemodynamics or BIS measurements.

Moreover, the problem of closed-loop control of the depth of analgesia (DoA) of a patient, similarly to the anesthesia, is a very challenging problem due to the numerous phenomena to be considered as patient variability, multivariable characteristics, positivity constraints, dynamics dependent on the hypnotic agent, ... Main attention in this domain was focused on anesthesia, although few works have concerned analgesia. Basically, PID-based feedback control strategies have been considered to adjust the amount of opioid administered [20]. Alternatively, the use of model predictive control was proposed in [10].

This paper revisits the control problem of the analgesic state of a patient under the framework of quantized [21] and saturated systems [18]. Actually, the output of the controlled system, which is the smallest magnitude of a stimulus that induces a significant dilatation of the pupil, is quantized, i.e, its value can only be a multiple of a constant. The

\footnotetext{
${ }^{1}$ LAAS-CNRS, Université de Toulouse, CNRS, Toulouse, France. \{szabi, queinnec, tarbour\}@laas.fr

${ }^{2} \mathrm{CHU}$ Toulouse, Département d'anesthésie-réanimation, 31059 cedex, France. mazerolles.mechu-toulouse.fr.

This work has been supported by ANR project LimICoS grant number 12 BS03 00501 .
}

global goal is then to control the DoA in an interval fixed a priori, taking into account directly the magnitude limitation of the control signal, that is the limitation of the rate of drug addition (in the current case the remifentanil) intravenously. Moreover, the dynamics of the evolution of the drug in the patient's body is usually described by a pharmacokinetic model with multiple time scales. Rather than treating the system as a singularly perturbed system [13], we reformulate the problem by separating fast and slow dynamics in order to reduce the global control problem to that one of the fast subsystem (the DoA being directly linked to the states of the fast subsystem) perturbed by the slow dynamics and vice versa. Finally, the main contribution of the paper resides in the control design of a dynamic output-feedback controller proposed through matrix inequalities conditions taking into account the quantized nature of the output and the saturation of the input.

\section{MODELling ASPECTS AND PROBLEM FORMULATION}

\section{A. The patient model}

The compartment model used to describe the circulation of drugs in a patient's body, also known as Pharmacokinetic/Pharmacodynamic (PK/PD) model, is based on a classical three-compartment model [8].

The effect of the drug on the patient is expressed throughout the effect site, which represents the action of drugs on the brain and is related to the concentration in the central compartment through a first order dynamic [4]. So, the compartmental model can be expressed as follows:

$$
\dot{x}_{a n}(t)=A x_{a n}(t)+B u_{a n}(t)
$$

with

$$
\begin{aligned}
A & =\left[\begin{array}{cccc}
-\left(k_{10}+k_{12}+a_{13}\right) & k_{21} & k_{31} & 0 \\
k_{12} & -k_{21} & 0 & 0 \\
k_{13} & 0 & -k_{31} & 0 \\
k_{e 0} / V_{1} & 0 & 0 & -k_{e 0}
\end{array}\right] \\
B & =\left[\begin{array}{llll}
1 & 0 & 0 & 0
\end{array}\right]^{\prime}
\end{aligned}
$$

where $x_{a n}=\left[\begin{array}{llll}x_{1} & x_{2} & x_{3} & x_{4}\end{array}\right]^{\prime}, x_{1}(t), x_{2}(t), x_{3}(t)$ are the masses in grams of the analgesic in the different compartments, $x_{4}(t)$ is the effect site concentration and $u_{a n}(t)$ is the infusion rate in $\mathrm{g} / \mathrm{min}$ of the analgesic. For numerical simulations, we use the Minto model [17], shown in Table I, to express the influence of the patient characteristics (weight, age, height, ...) on the parameters, but any other model could be used. The lean body mass (LBM) is calculated using the James formula [12]. 
TABLE I

MINTO MODEL PARAMETERS

\begin{tabular}{ll}
\hline Parameter & Estimation \\
\hline$V_{1}(L)$ & $5.1-0.0201($ age -40$)+0.072(L B M-55)$ \\
$V_{2}(L)$ & $9.82-0.0811($ age -40$)+0.108(L B M-55)$ \\
$V_{3}(L)$ & 5.42 \\
$k_{10}$ & {$[2.6-0.0162($ age -40$)+0.0191(L B M-55)] / V_{1}$} \\
$k_{12}$ & {$[2.05-0.0301($ age -40$)] / V_{1}$} \\
$k_{13}$ & {$[0.076-0.00113($ age -40$)] / V_{1}$} \\
$k_{21}$ & {$[2.05-0.0301($ age -40$)] / V_{2}$} \\
$k_{31}$ & {$[0.076-0.00113($ age -40$)] / V_{3}$} \\
$k_{e 0}$ & $0.595-0.007($ age -40$)$ \\
\hline
\end{tabular}

\section{B. Error model}

Consider $x_{e 4}$ the effect site concentration target corresponding to a desired DoA known. The other variables can then be deduced from the equilibrium point of system (1):

$$
x_{e 1}=x_{4 e} V_{1}, \quad x_{e 2}=\frac{k_{21}}{k_{12}} x_{e 1}, \quad x_{e 3}=\frac{a_{k 1}}{k_{13}} x_{e 1},
$$

and the value of the input for this equilibrium is given by

$$
u_{e}=k_{10} x_{e 1} .
$$

Finally, the error model is directly derived from system (1), considering the equilibrium point $x_{e}$ and the change of variable $x_{e r r}=x_{a n}-x_{e}, u_{e r r}=u_{a n}-u_{e}$. The positivity constraints on $u_{a n}$ and $x_{a n}$ can be expressed as constraints on $u_{e r r}$ and $x_{e r r}$ :

$$
\begin{aligned}
& x_{a n} \geq 0 \Rightarrow x_{e r r} \geq-x_{e} \\
& u_{a n} \geq 0 \Rightarrow u_{e r r} \geq-u_{e}
\end{aligned}
$$

Moreover, an upper limit on the amount of drugs that can be injected in the blood over time is also taken into account.

\section{The quantized measurement}

1) The DoA measurement: The clinical indicator used in this study is the pupil reaction to an external stimulus. Actually, the pupil size variation in reaction to an electrical pulse of given intensity is related to the effect site concentration of drug. This is illustrated on Figure 1 where the real pupil size variation of a patient (man, 50 years old, $110 \mathrm{~kg}, 180 \mathrm{~cm}$ ) is plotted in response to successive $40 \mathrm{~mA}$ impulse signals, for increasing effect site concentrations.

We then suggest a model, which relates the pupil size variation to the effect site concentration and the magnitude (in $\mathrm{mA}$ ) of the pulse stimulation signal, as follows:

$$
\left(\begin{array}{c}
\dot{x}_{5} \\
\dot{x}_{6}
\end{array}\right)=\left[\begin{array}{cc}
-k_{5} & 0 \\
k_{65}\left(1-\frac{x_{4}}{c_{\text {emax }}}\right) & -k_{6}
\end{array}\right]\left(\begin{array}{l}
x_{5} \\
x_{6}
\end{array}\right)+\left[\begin{array}{l}
1 \\
0
\end{array}\right] i m p
$$

where $x_{5}$ models the impulse signal imp as received by the human body and $x_{6}$ represents the pupil size variation in $\%$. This model illustrates that the more $x_{4}$ approaches $c_{\text {emax }}$, the less is the pupil reaction to an impulsive signal. The positive parameters $k_{5}, k_{6}, k_{65}$ and $c_{\text {emax }}$ are patient dependent and have to be identified, but this is outside the scope of the current paper. For an estimated set of parameters, this model response is illustrated in Figure 1 (in red).

Then, in order to use the information related to the pupil reaction to electrical stimuli, we define the output of the

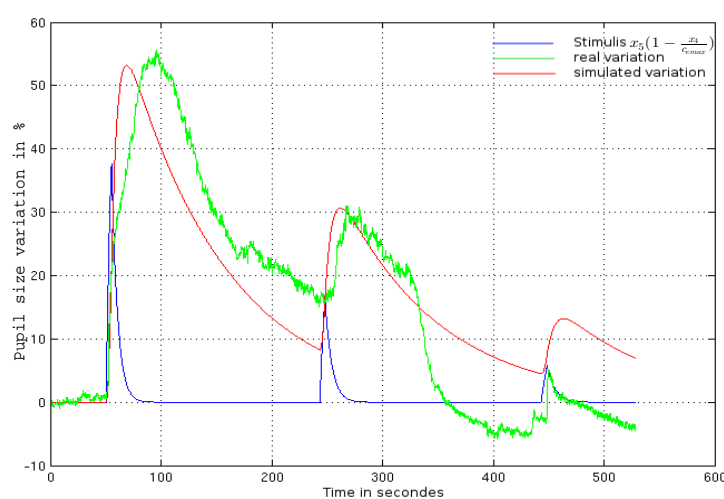

Fig. 1. Real and estimated pupil size variation in response to a $40 \mathrm{~mA}$ stimulus

PK/PD model as the smallest magnitude of stimuli that induces a significant (i.e. a reaction larger than a given threshold $\Theta$ ) pupil size variation. The output function $y_{i m p}=$ $h\left(x_{4}\right)$ may be derived from the manipulation of model (3), then linearized at the target setpoint (impulsion amplitude and/or effect site concentration) to obtain $y_{i m p}=c_{4} x_{4}$.

2) Quantization of the DoA: From a practical point of view, successive increasing impulsive pulses are delivered until the reaction size variation exceeds the threshold $\Theta$. This signifies that the output signal is a quantized signal. The quantization is defined around the equilibrium point established above, the output equation is then obtained by

$$
y=q\left(C x_{e r r}\right)
$$

with $C=\left[\begin{array}{cccc}0 & 0 & 0 & c_{4}\end{array}\right] \cdot q($.$) is the uniform quantizer,$ having as quantization error bound $\Delta>0$, defined by [9]

$$
q:\left\{\begin{array}{l}
\mathbb{R}^{p} \rightarrow \Delta \mathbb{Z}^{p} \\
z \mapsto \Delta \operatorname{sign}(z)\left\lfloor\frac{|z|}{\Delta}\right\rfloor
\end{array}\right.
$$

\section{Multiplicity of dynamics}

Furthermore, as discussed in [24], system (1) involves both fast and slow dynamics and the control action acts on the fast dynamics which is the key dynamics in the DoA control. Thus, thanks to the particular structure of the system, we split it into two subsystems, namely a fast subsystem (blood, effect site), on which acts the control input, and a slow subsystem (muscles and fat) whose dynamics is influenced only by the state of the fast subsystem. The slow subsystem is considered as a simple disturbance for the fast subsystem.

\section{E. Problem statement}

Let us denote $x_{f}=\left[\begin{array}{ll}x_{\text {err } 1} & x_{\text {err } 4}\end{array}\right]^{\prime} \in \mathbb{R}^{n}$ the fast state vector, $x_{s}=\left[\begin{array}{ll}x_{\text {err } 2} & x_{\text {err } 3}\end{array}\right]^{\prime} \in \mathbb{R}^{n}$ the slow state vector and $u=u_{\text {err }} \in \mathbb{R}^{m}$. The error system can be written as follows:

$$
\begin{aligned}
& \dot{x}_{f}=A_{f} x_{f}+A_{f s} x_{s}+B_{f} u \\
& \dot{x}_{s}=A_{s f} x_{f}+A_{s} x_{s} \\
& y_{f}=q\left(C_{f} x_{f}\right)
\end{aligned}
$$


with $y_{f} \in \mathbb{R}^{p}$. $q($.$) represents the quantizer given in (5).$ We consider a $n$-order dynamic output-feedback stabilizing controller with an anti-windup action of the form:

$$
\begin{aligned}
& \dot{x}_{c}=A_{c} x_{c}+B_{c} u_{c}+E_{c} \phi\left(y_{c}\right) \\
& y_{c}=C_{c} x_{c}+D_{c} u_{c}
\end{aligned}
$$

where $x_{c} \in \mathbb{R}^{n}, u_{c} \in \mathbb{R}^{p}, y_{c} \in \mathbb{R}^{m}$ are respectively the state, the input and the output of the controller. $\phi\left(y_{c}\right) \in \mathbb{R}^{m}$ is an input signal used to perform the anti-windup action introduced in order to reduce any undesirable effects caused by the actuator saturation. Matrices $A_{c}, B_{c}, C_{c}, D_{c}$ and $E_{c}$ are constant matrices of appropriate dimensions to be designed. The interconnection between the plant (6) and the controller (7) is done as follows:

$$
\begin{aligned}
& u=\operatorname{sat}\left(y_{c}\right) \\
& u_{c}=y_{f} \\
& \phi\left(y_{c}\right)=\operatorname{sat}\left(y_{c}\right)-y_{c}
\end{aligned}
$$

where sat(.) denotes the vector-valued saturation function defined as $\operatorname{sat}\left(y_{c(i)}\right)=\operatorname{sign}\left(y_{c(i)}\right) \min \left\{u_{0(i)},\left|y_{c(i)}\right|\right\}, i=$ $1, \ldots, m, u_{0(i)}>0$ being the level of saturation of the $i-t h$ component of $y_{c}$. According to (2), one sets the symmetric bound $u_{0}=u_{e}$.

Then, considering (6)-(7)-(8) yields the following closedloop system:

$$
\begin{aligned}
& \dot{x}_{f}=A_{f} x_{f}+B_{f} \operatorname{sat}\left(C_{c} x_{c}+D_{c} q\left(C_{f} x_{f}\right)\right)+A_{f s} x_{s} \\
& \dot{x}_{c}=A_{c} x_{c}+B_{c} q\left(C_{f} x_{f}\right)+E_{c} \phi\left(C_{c} x_{c}+D_{c} q\left(C_{f} x_{f}\right)\right) \\
& \dot{x}_{s}=A_{s} x_{s}+A_{s f} x_{f}
\end{aligned}
$$

By defining the augmented state $x=\left[\begin{array}{ll}x_{f}^{\prime} & x_{c}^{\prime}\end{array}\right]^{\prime} \in \mathbb{R}^{2 n}$ and by defining $\psi(z)=q(z)-z$, the closed-loop system (9) reads:

$$
\begin{aligned}
\dot{x}=\tilde{A} x+B_{\phi} \phi(K x & \left.+D_{c} \psi(\tilde{C} x)\right) \\
& +B_{\psi} \psi(\tilde{C} x)+B_{s} x_{s}
\end{aligned}
$$

with

$$
\begin{aligned}
& \tilde{A}=\left[\begin{array}{cc}
A_{f}+B_{f} D_{c} C_{f} & B_{f} C_{c} \\
B_{c} C_{f} & A_{c}
\end{array}\right] ; \quad B_{\phi}=\left[\begin{array}{c}
B_{f} \\
E_{c}
\end{array}\right] \\
& K=\left[\begin{array}{cc}
D_{c} C_{f} & C_{c}
\end{array}\right] ; \quad \tilde{C}=\left[\begin{array}{cc}
C_{f} & 0
\end{array}\right] \\
& B_{\psi}=\left[\begin{array}{c}
B_{f} D_{c} \\
B_{c}
\end{array}\right] ; \quad B_{s}=\left[\begin{array}{c}
A_{f s} \\
0
\end{array}\right] ; \quad B_{x}=\left[\begin{array}{ll}
A_{s f} & 0
\end{array}\right]
\end{aligned}
$$

The problem we intend to solve can then be formulated as follows:

Problem 1: Design $A_{c}, B_{c}, C_{c}, D_{c}, E_{c}$ and characterize three sets $\mathcal{A}_{0}, \mathcal{A}_{\infty}, \mathcal{A}_{s}$ such that:

1) the closed-loop trajectories of systems (10a) and (10b) are confined in the sets $\mathcal{A}_{0}$ and $\mathcal{A}_{s}$ respectively;

2) for any initial condition $x(0) \in \mathcal{A}_{0} \backslash \mathcal{A}_{\infty}$, the trajectories of system (10a) converges to $\mathcal{A}_{\infty} \subset \mathcal{A}_{0}$.

Remark 1: Notice that, due to the presence of the uniform quantizer, the right-hand side of equation (10a) is discontinuous. Then there is no guarantee about the existence of solutions in a classical sense [6]. The notion of solution should be then properly defined. To this end, similarly to
[21], we suppose that the Caratheodory solutions for the system (10a) exist. Another option would be to consider Krasovskii solutions [9].

\section{CONTROller SYNTHESIS}

\section{A. Main results}

The closed-loop system (10) contains nested nonlinearities since $\phi$ depends on $\psi$. To solve Problem 1, we exploit the sector conditions both for $\psi$ as presented in [9] and for $\phi$ as given in [22]. Let us recall these conditions.

Lemma 1: [9] Let $z \in \mathbb{R}^{p}$, and let $S_{1}, S_{2} \in \mathbb{R}^{p \times p}$ be diagonal positive definite matrices. Then the pair $(\psi(z), z)$ satisfies the following conditions:

$$
\begin{aligned}
& \psi^{\prime}(z) S_{1} \psi(z)-\operatorname{trace}\left(S_{1}\right) \Delta^{2} \leq 0 \\
& \psi^{\prime}(z) S_{2}(\psi(z)+z) \leq 0
\end{aligned}
$$

Lemma 2: [22] Considering a matrix $G \in \mathbb{R}^{m \times 2 n}$, the non-linearity $\phi\left(y_{c}\right)$ satisfies

$$
\phi\left(y_{c}\right)^{\prime} T\left(\phi\left(y_{c}\right)+D_{c} \psi(\tilde{C} x)+G x\right) \leq 0
$$

for any diagonal positive matrix $T \in \mathbb{R}^{m \times m}$ if $x \in \mathcal{S}\left(u_{0}\right)$ defined by

$$
\mathcal{S}\left(u_{0}\right)=\left\{\begin{array}{c}
x \in \mathbb{R}^{n} ; \forall i \in\{1, \ldots, m\} \\
-u_{0(i)} \leq\left(K_{(i)}-G_{(i)}\right) x \leq u_{0(i)}
\end{array}\right\}
$$

Now, we are in position to state the main result to address Problem 1.

Theorem 1: If there exist two symmetric positive definite matrices $P \in \mathbb{R}^{2 n \times 2 n}, Q \in \mathbb{R}^{n \times n}$, three diagonal positive definite matrices $S_{1}, S_{2} \in \mathbb{R}^{p \times p}, T \in \mathbb{R}^{m \times m}$, a matrix $G \in$ $\mathbb{R}^{m \times 2 n}$, matrices $A_{c}, B_{c}, C_{c}, D_{c}, E_{c}$ and positive scalars $\tau_{1}, \tau_{2}, \tau_{3}, \tau_{4}, \tau_{5}, \eta, \delta$ such that ${ }^{1}$

$$
\left[\begin{array}{cccc}
H e(P \tilde{A})+\left(\tau_{1}-\tau_{2}\right) P & P B_{\psi}-\tilde{C}^{\prime} S_{2} & P B_{\phi}-G^{\prime} T & P B_{s} \\
\star & -S_{1}-2 S_{2} & -D_{c}^{\prime} T & 0 \\
\star & \star & -2 T & 0 \\
\star & \star & \star^{\prime} & -\tau_{3} Q
\end{array}\right]<0
$$

$$
\begin{gathered}
{\left[\begin{array}{cc}
H e\left(Q A_{s}\right)+\tau_{4} Q & Q B_{x} \\
\star & -\tau_{5} P
\end{array}\right]<0} \\
{\left[\begin{array}{cc}
P & K_{(i)}^{\prime}-G_{(i)}^{\prime} \\
\star & \eta u_{0(i)}^{2}
\end{array}\right] \geq 0 i=1, \ldots, m .} \\
\eta\left(\tau_{3}+\delta\left(\Delta^{2} \operatorname{trace}\left(S_{1}\right)-\tau_{1}\right)\right)+\tau_{2} \delta \leq 0 \\
\eta \leq 1 \\
-\tau_{4} \eta+\tau_{5} \delta \leq 0
\end{gathered}
$$

then

$$
\begin{gathered}
A_{c}, B_{c}, C_{c}, D_{c}, E_{c} \\
\mathcal{A}_{0}=\mathcal{E}(P, \eta):=\left\{x \in \mathbb{R}^{2 n}: x^{\prime} P x \leq \eta^{-1}\right\} \\
\mathcal{A}_{\infty}=\mathcal{E}(P):=\left\{x \in \mathbb{R}^{2 n}: x^{\prime} P x \leq 1\right\}
\end{gathered}
$$

\footnotetext{
${ }^{1}$ In symmetric matrices, the notation $\star$ stands for symmetric blocks and $\operatorname{He}(A)=A^{\prime}+A$.
} 


$$
\mathcal{A}_{s}=\mathcal{E}(Q):=\left\{x_{s} \in \mathbb{R}^{n}: x_{s}^{\prime} Q x_{s} \leq \delta^{-1}\right\}
$$

are solution to Problem 1.

Proof: One has to prove that the trajectories of subsystem (10a) remain confined in a compact set $\mathcal{E}(P, \eta)$ as long as the trajectories of subsystem $(10 \mathrm{~b})$ are in $\mathcal{E}(Q, \delta)$. Furthermore, one has also to prove that the trajectories of the closed-loop system (10a) initialized in $\mathcal{E}(P, \eta)$ converge to a compact set $\mathcal{E}(P) \subset \mathcal{E}(P, \eta)$.

Consider, for the closed-loop fast subsystem (10a), the quadratic Lyapunov function $V(x)=x^{\prime} P x, P=P^{\prime}>0$. One has then to prove that $\dot{V}(x)<-\alpha(V(x)), \alpha$ being a $\mathcal{K}$-function, for any $x$ such that $x^{\prime} P x \leq \eta^{-1}$ and $x^{\prime} P x \geq 1$, and for any $x_{s} \in \mathcal{E}(Q, \delta)$. In other words, we have to verify by using the $S$-procedure the following inequality:

$$
\begin{gathered}
\dot{V}(x)+\tau_{1}\left(x^{\prime} P x-1\right)+\tau_{2}\left(\eta^{-1}-x^{\prime} P x\right) \\
+\tau_{3}\left(\delta^{-1}-x_{s}^{\prime} Q x_{s}\right)<-\alpha(V(x))
\end{gathered}
$$

Furthermore, by using Lemmas 1 and 2, a sufficient condition to verify (21) is that

$$
\begin{aligned}
& \dot{V}(x)+\tau_{1} x^{\prime} P x-\tau_{2} x^{\prime} P x-\tau_{3} x_{s}^{\prime} Q x_{s}-\psi^{\prime} S_{1} \psi \\
& -2 \psi^{\prime} S_{1}(\psi+\tilde{C} x)-2 \phi^{\prime} T\left(\phi+D_{c} \psi+G x\right)<-\alpha(V(x))
\end{aligned}
$$

and

$$
\operatorname{trace}\left(S_{1}\right) \Delta^{2}-\tau_{1}+\tau_{2} \eta^{-1}+\tau_{3} \delta^{-1}<0
$$

as long as $\mathcal{E}(P, \eta) \subseteq S\left(u_{0}\right)$, which is ensured by satisfying inequality (17). The inequality (22) can be written as $\zeta^{\prime} \mathcal{L} \zeta<$

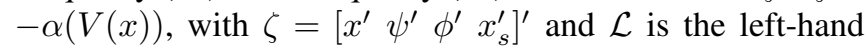
side matrix of inequality (15). Hence, the satisfaction of inequalities (15) and (18) means that it exists a small enough positive scalar $\alpha$ such that $\dot{V}(x)+\tau_{1}\left(x^{\prime} P x-1\right)-\tau_{2}\left(x^{\prime} P x-\right.$ $\left.\eta^{-1}\right)+\tau_{3}\left(\delta^{-1}-x_{s}^{\prime} Q x_{s}\right) \leq-\alpha x^{\prime} x$ which in turn gives (21). Moreover, one has to prove that the set $\mathcal{A}_{0}=\mathcal{E}(P, \eta)$ contains the set $\mathcal{A}_{\infty}=\mathcal{E}(P)$ which holds if $\eta \leq 1$.

On the other hand, similarly to [24], [18], the satisfaction of relations (16) and (20) ensures the invariance of the ellipsoid $\mathcal{E}(Q, \delta)$ for the subsystem (10b), for any $x \in \mathcal{E}(P, \eta)$.

Remark 2: The fact that the set $\mathcal{E}(P)$ is an attractor for the trajectories of system (10) means that, inside this set, $x_{f}$ and $x_{c}$ evolve close to the origin. Then, considering only the $x_{f}$ part, that means that $x_{1}, x_{4}$ evolve close to their equilibrium points $x_{1 e}, x_{4 e}$. However, it is important to point out that, without additional conditions, we cannot ensure the convergence of $x_{1}, x_{4}$ to $x_{1 e}, x_{4 e}$. Indeed, in general, the asymptotic stability properties of the quantization-free closed-loop system are destroyed by quantization [9] and the implicit interest by minimizing the size of the set $\mathcal{E}(P)$ is to ensure that $x_{1}, x_{4}$ will remain close to their equilibrium point.

Remark 3: Note that the approach induces several sources of conservatism (these are only sufficient conditions) and in particular, it assumes that the disturbance (the slow dynamics) may evolve randomly in $\mathcal{A}_{s}$, not considering the fact that it is actually a vanishing disturbance.

\section{B. Computational issue}

Theorem 1 provides a sufficient condition in terms of matrix inequalities to solve Problem 1. Nevertheless, some matrix inequalities of Theorem 1 are nonlinear since having some products between decision variables, in particular involving the matrices $A_{c}, B_{c}, C_{c}, D_{c}$ and $E_{c}$ and the Lyapunov matrix $P$. Then, these conditions are hardly tractable from a numerical point of view, making in general impossible to design a suitable controller directly from Theorem 1 . In order to partly linearize the conditions of Theorem 1, we use the congruence transformation proposed in [19] to propose a sufficient set of conditions to solve Problem 1.

Proposition 1: If there exist three symmetric positive definite matrices $X, Y, Q \in \mathbb{R}^{n \times n}$, three diagonal positive definite matrices $S_{1}, S_{2} \in \mathbb{R}^{p \times p}, S \in \mathbb{R}^{m \times m}$, matrices $W \in$ $\mathbb{R}^{n \times n}, R \in \mathbb{R}^{n \times m}, L \in \mathbb{R}^{n \times p}, M \in \mathbb{R}^{m \times n}, N \in \mathbb{R}^{m \times p}$, $Z \in \mathbb{R}^{m \times n}, Z_{1} \in \mathbb{R}^{m \times n}$ and positive scalars $\tau_{1}, \tau_{2}, \tau_{3}, \tau_{4}$, $\tau_{5}, \eta, \delta$ such that,

$\left[\begin{array}{cccc}H e\left(H_{1}\right)+\left(\tau_{1}-\tau_{2}\right) H_{2} & H_{3} & H_{6} & H_{4} \\ \star & -S_{1}-2 S_{2} & -N^{\prime} & 0 \\ \star & \star & -2 S & 0 \\ \star & \star & \star & -\tau_{3} Q\end{array}\right]<0$

$$
\begin{gathered}
{\left[\begin{array}{cc}
H e\left(Q A_{s}\right)+\tau_{4} Q & Q H_{5} \\
\star & -\tau_{5} H_{2}
\end{array}\right]<0} \\
{\left[\begin{array}{cc}
H_{2} & H_{7} \\
\star & \eta u_{0(i)}^{2}
\end{array}\right] \geq 0 i=1, \ldots, m}
\end{gathered}
$$

and scalar conditions (18), (19) and (20), where

$$
\begin{aligned}
& H_{1}=\left[\begin{array}{cc}
A_{f} Y+B_{f} M & A_{f}+B_{f} N C_{f} \\
W & X A_{f}+L C_{f}
\end{array}\right] \\
& H_{2}=\left[\begin{array}{cc}
Y & I \\
\star & X
\end{array}\right], H_{3}=\left[\begin{array}{c}
B_{f} N-Y C_{f}^{\prime} S_{2} \\
L-C_{f}^{\prime} S_{2}
\end{array}\right] \\
& H_{4}=\left[\begin{array}{c}
A_{f s} \\
X A_{f s}
\end{array}\right], H_{5}=\left[\begin{array}{ll}
A_{s f} Y & A_{s f}
\end{array}\right] \\
& H_{6}= {\left[\begin{array}{c}
B_{f} S-Z^{\prime} \\
R-Z_{1}^{\prime}
\end{array}\right], H_{7}=\left[\begin{array}{c}
M_{(i)}{ }^{\prime}-Z_{(i)}{ }^{\prime} \\
C_{f}^{\prime} N_{(i)}{ }^{\prime}-Z_{1(i)}
\end{array}\right] } \\
& i=1, \ldots, m .
\end{aligned}
$$

then

$$
\begin{aligned}
& E_{c}=U^{-1}\left(R-X B_{f} S\right) S^{-1} \\
& D_{c}=N \\
& C_{c}=\left(M-N C_{f} Y\right)\left(V^{\prime}\right)^{-1} \\
& B_{c}=U^{-1}\left(L-X B_{f} N\right) \\
& A_{c}=U^{-1}\left(W-X A_{f} Y-X B_{f} M-U B_{c} C_{f} Y\right)\left(V^{\prime}\right)^{-1}
\end{aligned}
$$

and $\mathcal{A}_{0}=\mathcal{E}(P, \eta):=\left\{x \in \mathbb{R}^{2 n}: x^{\prime} P x \leq \eta^{-1}\right\}$, $\mathcal{A}_{\infty}=\mathcal{E}(P):=\left\{x \in \mathbb{R}^{2 n}: x^{\prime} P x \leq 1\right\}, \mathcal{A}_{s}=\left\{x_{s} \in\right.$ 
$\left.\mathbb{R}^{n}: x_{s}^{\prime} Q x_{s} \leq \delta^{-1}\right\}$ where $U, V \in \mathbb{R}^{n}$ are any nonsingular matrices such that $U V^{\prime}=I-X Y$ and

$$
\left.P=\left[\begin{array}{cc}
X & U \\
U^{\prime} & \hat{X}
\end{array}\right] \text { with } \hat{X}=U^{\prime}\left(X-Y^{-1}\right)^{-1}\right) U
$$

are solution to problem 1 .

Proof: Considering the matrix $P$ defined in (27), one can define

$$
\mathbb{J}=\left[\begin{array}{cc}
Y & V \\
I & 0
\end{array}\right]
$$

which is a nonsingular matrix as $U$ and $V$ are assumed to be nonsingular. The objective is to rearrange the conditions of Theorem 1 thanks to adequate changes of variables. Then, by pre- and post-multiplying condition (15) by $\operatorname{diag}\{\mathbb{J}, I, S, I\}$ and $\operatorname{diag}\left\{\mathbb{J}^{\prime}, I, S, I\right\}$, with $S=T^{-1}$, one gets

$$
\left[\begin{array}{cccc}
H e\left(\mathbb{D} P \tilde{A} \mathbb{J}^{\prime}\right)+\left(\tau_{1}-\tau_{2}\right) \mathbb{J} P \mathbb{J}^{\prime} & \star & \star & \star \\
B_{\psi}^{\prime} P \mathbb{J}^{\prime}-S_{2} \tilde{C} \mathbb{J}^{\prime} & -S_{1}-2 S_{2} & \star & \star \\
S B_{\phi}^{\prime} P \mathbb{J}^{\prime}-G \mathbb{J}^{\prime} & -D_{c} & -2 S & \star \\
B_{s} P \mathbb{J}^{\prime} & 0 & 0 & -\tau_{3} Q
\end{array}\right]
$$

Let us consider the following changes of variables

$$
\begin{aligned}
& {\left[\begin{array}{cc}
W & L \\
M & N
\end{array}\right]=\left[\begin{array}{cc}
X A_{f} Y & 0 \\
\star & 0
\end{array}\right]+} \\
& {\left[\begin{array}{cc}
U & X B_{f} \\
0 & I
\end{array}\right]\left[\begin{array}{cc}
A_{c} & B_{c} \\
C_{c} & D_{c}
\end{array}\right]\left[\begin{array}{cc}
V^{\prime} & 0 \\
C_{f} Y & I
\end{array}\right], } \\
Z_{1}= & G_{1}, Z=G_{1} Y+G_{2} V^{\prime}, R=X B_{f} S+U E_{c} S .
\end{aligned}
$$

with $G=\left[\begin{array}{ll}G_{1} & G_{2}\end{array}\right]$. It turns out, after calculations, that $\mathbb{J} P \tilde{A} \mathbb{J}^{\prime}=H_{1}, \mathbb{J} P \mathbb{J}^{\prime}=H_{2}, \mathbb{J P} B_{\psi}-\mathbb{J} \tilde{C}^{\prime} S_{2}=$ $H_{3}, \mathbb{J} P B_{\phi} S-\mathbb{J} G^{\prime}=H_{6}$ and $\mathbb{J} P B_{s}=H_{4}$. Then, one obtains relation (24). By the same way, relation (25) is obtained by pre- and post-multiplying (16) by $\operatorname{diag}\{I, \mathbb{J}\}$ and $\operatorname{diag}\left\{I, \mathbb{J}^{\prime}\right\}$, by noting that $B_{x} \Xi^{\prime}=H_{5}$. Relation (26) is obtained by pre- and post-multiplying (17) $\operatorname{diag}\{\mathbb{J}, I\}$ and $\operatorname{diag}\left\{\mathbb{J}^{\prime}, I\right\}$ and by noting $\mathbb{J}\left(K_{(i)}^{\prime}-G_{(i)}^{\prime}\right)=H_{7}$.

\section{Optimization issue}

The implicit objective behind Problem 1 is to obtain a set $\mathcal{A}_{0}$ as large as possible with respect to some practical constraints and a set $\mathcal{A}_{\infty}$ as small as possible. Those sets are characterized by the same matrix $P$ and the distance between the two sets is directly related to $\eta$. Then we can consider an optimization problem minimizing both $\operatorname{trace}\left(P^{-1}\right)$ and $\eta$ (see, for example, [5] or [18]). From the definition of $P$ in (27), it follows that:

$$
P^{-1}=\left[\begin{array}{cc}
Y & V \\
V^{\prime} & \hat{Y}
\end{array}\right]
$$

with $\hat{Y}=-U^{-1} X(I-X Y)^{\prime} U^{\prime-1}$. Matrix $U$ may be arbitrary chosen as any nonsingular matrix without any influence on the feasibility of conditions given in Proposition 1. In particular, by selecting $U=X$, it follows: $\operatorname{trace}\left(P^{-1}\right)=$
$\operatorname{trace}(Y)+\operatorname{trace}(\hat{Y})=2 \operatorname{trace}(Y)-\operatorname{trace}\left(X^{-1}\right)$. Then, the considered optimization problem consists in

$$
\min \operatorname{trace}(X+Y)+\eta
$$

Practical constraints relative to the set $\mathcal{A}_{0}$ are suggested to guarantee that the DoA remains in the range $[-l \Delta,+l \Delta], l$ being an integer to be chosen, corresponding to some degree of freedom. This constraint can be represented by:

$$
\mathcal{P}=\left\{x \in \Re^{2 n}:\left|a_{k}^{\prime} x\right| \leq 1\right\}
$$

with $a_{k}^{\prime}=\left[\begin{array}{llll}0 & l / \Delta & 0 & 0\end{array}\right]$. The inclusion of $\mathcal{A}_{0}$ in $\mathcal{P}$ reads

$$
\left[\begin{array}{cc}
P & a_{k} \\
\star & \eta
\end{array}\right] \geq 0
$$

and, by pre- and post-multiplying this inequality by $\operatorname{diag}\{\mathbb{J}, 1\}$ and $\operatorname{diag}\left\{\mathbb{J}^{\prime}, 1\right\}$, it follows:

$$
\left[\begin{array}{cc}
H_{2} & H_{8} \\
\star & \eta
\end{array}\right] \geq 0
$$

with $H_{8}=\rrbracket a_{k}=\left[\begin{array}{ll}a_{k 1}^{\prime} Y & a_{k 1}^{\prime}\end{array}\right]^{\prime}, a_{k 1}^{\prime}=\left[\begin{array}{ll}0 & l / \Delta\end{array}\right]$.

Moreover, the relations of Proposition 1 yet involve some products between decision variables, in particular in the terms $\left(\tau_{1}-\tau_{2}\right) H_{2}, \tau_{3} Q, \tau_{4} Q, \tau_{5} H_{2}, Y C_{f}^{\prime} S_{2}, Q H_{5}$ in (24) and (25), or still $\eta\left(\tau_{3}-\tau_{1}\right), \eta \operatorname{trace}\left(S_{1}\right)$ in (18) and $\tau_{4} \eta$ in (20). Nevertheless, by fixing $\tau_{1}, \tau_{2}, \tau_{3}, \tau_{4}, \tau_{5}, \delta, S_{1}, S_{2}$ and $Q$, the relations become linear. Hence, except for $Q$, they can be selected by performing a grid search over a certain interval. Regarding the $Q H_{5}$ term, one can proceed in three steps. First, by considering any symmetric positive matrix $Q_{0}$ (corresponding to some given perturbation set), we compute a solution to the optimization problem (29) with respect to the LMI conditions of the fast system only (18), (19), (24) and (26). Secondly, considering $X, Y$ solution to the first step, search for the largest invariant set $\mathcal{E}(Q) \subset \mathcal{E}\left(Q_{0}\right)$ that satisfies the LMIs (20) and (25) associated to the slow subsystem only. Finally, take the matrix $Q$ solution to the second step and solve the full optimization problem:

$$
\begin{aligned}
& \min _{X, Y, S, L, W, R, M, N, Z, Z_{1}, \eta} \operatorname{trace}(X+Y)+\eta \\
& \text { subject to }(24)-(26),(18)-(20),(30)
\end{aligned}
$$

\section{NUMERICAL ILLUSTRATION}

Consider a nominal patient, man, 53 years old, $77 \mathrm{~kg}$ and $177 \mathrm{~cm}$. By considering the quantization error $\Delta=1$ which means that the DoA is given as multiple of $1 \mathrm{~mA}$ and setting $\tau_{1}=1.23, \tau_{2}=0.1, \tau_{3}=0.3, \tau_{4}=0.02, \tau_{5}=0.0485$, $\delta=0.3306 ; S_{1}=0.3225$ and $S_{2}=0.002$, the following controller, solution to Problem 1 is obtained:

$\left[\begin{array}{c|c}A_{c} & B_{c} \\ \hline C_{c} & D_{c}\end{array}\right]=\left[\begin{array}{cc|c}-24.78 & -29.19 & 0.18 \\ -0.38 & -3.15 & -0.14 \\ \hline 22.88 & 19.34 & -0.78\end{array}\right], E_{c}=\left[\begin{array}{c}-0.11 \\ 0.08\end{array}\right]$

The initial state (no drug in the patient) is associated to a DoA $=12.5 \mathrm{~mA}$, which corresponds to a patient awake (pupil of a patient awake does not react for small stimulation). The target reference is then set to $40 \mathrm{~mA}$, with the objective to achieve a target interval $[-5 \Delta,+5 \Delta]$. Figures 2 and 3 
show the evolution of the DoA and of the drug infusion rate, respectively. One can check in Figure 2 that the time response to reach the target interval is about 4.3 minutes.

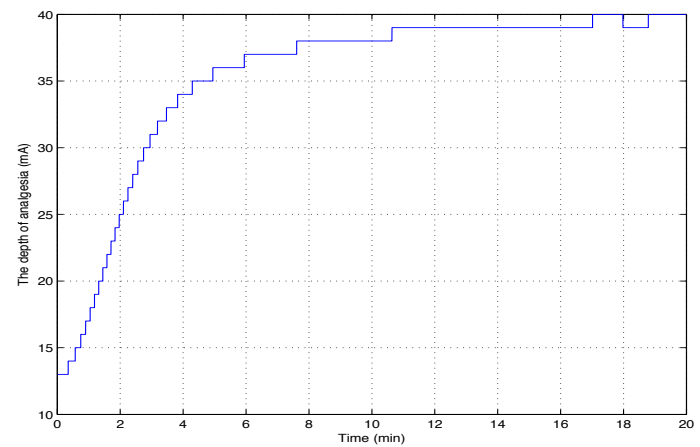

Fig. 2. DoA evolution ( $\left.y_{i m p}\right)$

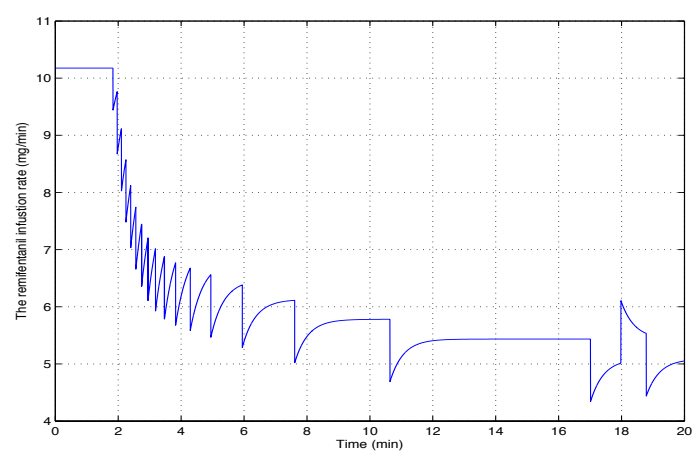

Fig. 3. Drug infusion rate $\left(u_{a n}=u_{e}+\operatorname{sat}\left(y_{c}\right)\right)$

\section{CONCLUSION}

The novelty of this work is that, with the introduction of a new analgesia indicator, we presented a dynamic output feedback controller for a decoupled model taking into account the output quantization and the input saturation. The decomposition of the model into a fast and a slow systems allows to focus the control design on the fast subsystem, whereas the slow one is considered as a disturbance. For the future, the fact that the DoA information is actually a sampled output, obtained only by performing pulses on the patient, will have to be taken into account. Moreover, the synthesized controller guarantees to keep the DoA in the range $[-\Delta,+\Delta]$ but does not address the induction phase corresponding to the first drug injection produced by the anesthetist. To finalize the automation of the entire process, the next step will be to propose a switched control law inspired by the practice.

\section{REFERENCES}

[1] J. M. Bailey and W. M. Haddad. Drug dosing control in clinical pharmacology. IEEE Control Systems Magazine, 25(2):35-51, 2005.

[2] R. Balocchi, M. Varanini, D. Menicucci, E.L. Santarcangelo, S. Migliorini, G. Fontani, and G. Carli. Heart rate variability in subjects with different hypnotic susceptibility receiving nociceptive stimulation and suggestions of analgesia. In Annual International Conference of the IEEE Engineering in Medicine and Biology Society (EMBS), volume 7, pages 6996-6999, Shanghai, China, 2005.
[3] L. Barvais, E. Engelman, J. M. Eba, E. Coussaert, F. Cantraine, and G. N. Kenny. Effect site concentrations of remifentanil and pupil response to noxious stimulation. British Journal of Anaesthesia, 91(3):347-352, 2003.

[4] C. L. Beck. Modeling and control of pharmacodynamics. European Journal of Control, 24:33-49, 2015.

[5] S. Boyd, L. El Ghaoui, E. Feron, and V. Balakrishnan. Linear Matrix Inequalities In System And Control Theory. Siam Philadelphia, 1994.

[6] J. Cortes. Discontinuous dynamical systems. Control Systems, IEEE, 28(3):36-73, June 2008.

[7] J. De Jonckheere, D. Rommel, J. Nandrino, M. Jeanne, and R. Logier. Heart rate variability analysis as an index of emotion regulation processes: Interest of the Analgesia Nociception Index (ANI). In Annual International Conference of the IEEE Engineering in Medicine and Biology Society (EMBS), pages 3432-3435, San Diego, USA, 2012.

[8] H. Derendorf and B. Meibohm. Modeling of pharmacokinetic / pharmacodynamic $(\mathrm{pk} / \mathrm{pd})$ relationships: Concepts and perspectives. Pharmaceutical Research, 16(2):176-185, 1999.

[9] F. Ferrante, F. Gouaisbaut, and S. Tarbouriech. Stabilization of continuous-time linear systems subject to input quantization. Automatica, 58:167-172, 2015

[10] A. Gentilini, C. Schaniel, M. Morari, C. Bieniok, R. Wymann, and T. Schnider. A new paradigm for the closed-loop intraoperative administration of analgesics in humans. IEEE Transactions on Biomedical Engineering, 49(4):289-299, 2002.

[11] M Huiku, K. Uutela, M. van Gils, I. Korhonen, M. Kymäläinen, P. Meriläinen, M. Paloheimo, M. Rantanen, P. Takala, H. Viertiö-Oja, and A. Yli-Hankala. Assessment of surgical stress during general anaesthesia. British journal of anaesthesia, 98:447-455, 2007.

[12] W. James. Research on obesity. Her majesty's stationary office, 1976.

[13] P. V. Kokotovic, H. K. Khalil, and J. O'Reilly. Singular perturbation methods in control: analysis and design, volume 29. New York: Academic, 1987.

[14] J. M. Lemos, D. V. Caiado, B. A. Costa, L. A. Paz, T. F. Mendonca, S. Esteves, and M. Seabra. Robust control of maintenance-phase anesthesia. IEEE Control Systems Magazine, 34(6):24-38, December 2014

[15] D. M. Mathews, P. M. Cirullo, M. M. R. F. Struys, T. De Smet, R. J. Malik, C. L. Chang, and G. G. Neuman. Feasibility study for the administration of remifentanil based on the difference between response entropy and state entropy. British Journal of Anaesthesia, 98:785-791, 2007.

[16] M. Mazerolles. La pupillométrie permet-elle de mesurer la profondeur d'anesthésie ? Praticien en Anesthésie Réanimation, 13(2):109-115, 2009 (in french).

[17] C. F. Minto, T. W. Schneider, and S. L. Shafer. Pharmacokinetics and pharmacodynamics of remifentanil.Model application. Anesthesiology, 86(1):24-33, 1997.

[18] S. Tarbouriech, G. Garcia, J. M. Gomes da Silva Jr. and I. Queinnec and S Tarbouriech. Stability and Stabilization of Linear Systems with Saturating Actuators. Springer, 2011.

[19] C. Scherer, P. Gahinet, and M. Chilali. Multiobjective output-feedback control via LMI optimization. IEEE Transactions on Automatic Control, 42:896-911, 1997.

[20] K. Soltesz, Ji. O. Hahn, G. A. Dumont, and J. M. Ansermino. Individualized PID control of depth of anesthesia based on patient model identification during the induction phase of anesthesia. In IEEE Conference on Decision and Control, pages 855-860, Orlando, USA, 2011

[21] S. Tarbouriech and F. Gouaisbaut. Control design for quantized linear systems with saturations. IEEE Transactions on Automatic Control, 57:1883-1889, 2012

[22] S. Tarbouriech, C. Prieur, and J. M. Gomes da Silva Jr. Stability analysis and stabilization of systems presenting nested saturations. IEEE Transactions on Automatic Control, 51(8):1364-1371, 2006.

[23] T. Ushiyama, K. Mizushige, H. Wakabayashi, T. Nakatsu, K. Ishimura, Y. Tsuboi, H. Maeta, and Y. Suzuki. Analysis of heart rate variability as an index of noncardiac surgical stress. Heart and Vessels, 23:53-59, 2008

[24] S. Zabi, I. Queinnec, S. Tarbouriech, G. Garcia, and M. Mazerolles. New approach for the control of anesthesia based on dynamics decoupling. In 9th IFAC Symposium on Biological and Medical Systems (BMS 2015), Berlin, Germany, 2015. 